\title{
Adiabatic Motions of Charged Particles in a Dipole Model of the Magnetosphere ${ }^{\dagger}$
}

\author{
Edward W. HONEs, JR.* \\ Department of Physics and Astronomy, University of Iowa, Iowa City, Iowa
}

\begin{abstract}
The use of the first and second adiabatic invariants of charged particle motion in calculating the trajectories of particles drifting in combined magnetic and electric fields is discussed. Such calculations become particularly simple for a dipole magnetic field if the magnetic lines of force are electric equipotentials,
\end{abstract}

\section{Introduction}

The second, or integral invariant of charged particle motion in a magnetic field with mirror-geometry has found wide use in studies of natural and artificial radiation belts around the earth. It has provided an indispensable tool for organizing the measurements of trapped particles by rockets and earth satellites and even for correlating such measurements with groundbased and balloon observations of various phenomena associated with precipitation of particles from the radiation belts. The integral invariant is useful in this important application, however, only in dealing with particles whose kinetic energy is great enough that their drift motions consist solely or largely of magnetic (gradient and line curvature) drifts, with electric fields producing negligible drift motions. Knowledge of the electric fields in the magnetosphere is incomplete. However, there are for example, those which drive the $S_{q}$ and $D_{s}$ current systems and these appear large enough to limit simple ordering of trapped particle measurements to particles with kinetic energy greater than 30 or $40 \mathrm{keV}$.

The existence of an integral invariant is not limited to situations in which particle drift motions consist solely of magnetic drifts but applies more generally to situations in which electric fields influence the particle motion. Northrop and Teller [1960] show that in the general case where electric fields (both conservative and non-conservative)act upon particles in a magnetic field, the guiding center motion of the particles will be such as to conserve the particles' magnetic moment, integral invariant, and total (kinetic plus potential) energy.

This paper examines the use of these conservation laws to determine the paths of charged particules through combined magnetic and electric fields, applying them specifically (as an example) to the determination of particle motions in the inner portions of the earth's magnetosphere where the magnetic field approximates that of a dipole. The electric field is assumed, in this example to consist of that caused by the earth's rotation plus that caused by the solar daily tidal action in the ionosphere.

* On leave of absence from the Institute for Defense Analyses, Washington, D.C.

$\dagger$ Research supported in part by the National Aeronautics and Space Administration under Grant NsG$233-62$, 


\section{The Integral Invariant in a Dipole Magnetic Field Whose Lines of Force are Electric Equipotentials}

The integral invariant, $J$, of particle motion is :

$$
J=\oint p_{\|} \mathrm{d} s=\oint \sqrt{2 m W_{\|}} \mathrm{d} s
$$

With conservation of a particle's magneticmoment, $M=\frac{W_{\perp}}{B}=\frac{W}{B_{m}}$, and with the assumption that magnetic lines of force are electric equipotenitals, equation (1) can be written :

$$
J=\sqrt{2 m M} \sqrt{B_{m}} \oint\left(1-B / B_{m}\right)^{1 / 2} \mathrm{~d} s
$$

In equations (1) and (2)

$$
p_{11}=\text { component of a particle's momentum parallel to magnetic line of force }
$$

$m=$ particle mass

$W=$ particle's kinetic energy with $W_{\perp}$ and $W_{\|}$referring to the amounts of kinetic energy associated with the particle's velocity components perpendicular and parallel to the magnetic line of force

$B=$ magnetic field strength

$B_{m}=$ magnetic field strength at which the particle will mirror

$\oint \mathrm{d} s=$ integral along a line of force between two successive mirrorings of a particle

The term $\sqrt{B_{m}}$ has been removed from the integral sign because a particle's kinetic energy and thus its mirror point $\left(B_{m}\right)$ will not change in a single bounce along a line of force (assumed an equipotential).

In the complete absence of significant electric fields, a particle's kinetic energy (and thus $B_{m}$ ) remains constant not just over a single bounce period, but over any number of bounce periods. In that event the particle's drift takes place along the surface on which the quantity, $\oint\left(1-B / B_{m}\right)^{1 / 2} \mathrm{ds}$ is constant; this is the basis of the parameter, $L$, defined by McIlwain [1961] and used so successfully to organize measurements of high-energy trapped particles. However, in the presence of significant electric fields, $W$ (and therefore $B_{m}$ ) will change as the particle moves from line to line, and the particle's drift will then be along the surface on which

$$
J^{\prime}=\frac{J}{\sqrt{2 m M}}=\sqrt{B_{m}} \oint\left(1-B / B_{m}\right)^{1 / 2} \mathrm{~d} s
$$

is constant.

Figure 1 depicts the lines of force and contours of constant field strength in a dipole magnetic field representing that of the earth. Also indicated are contours of constant $J^{\prime}$,

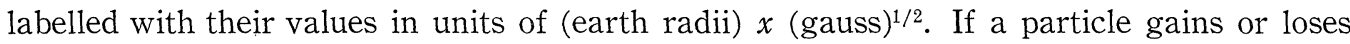
kinetic energy as it moves through the field, its mirror point will move inward or outward, respectively, along the surface of constant $J^{\prime}$ which contains its initial mirror point.

If the particle initially mirrors in the equatorial plane, $J^{\prime}=0$ and the particle must continue to mirror in the equatorial plane. In this case, the particle's kinetic energy is simply 


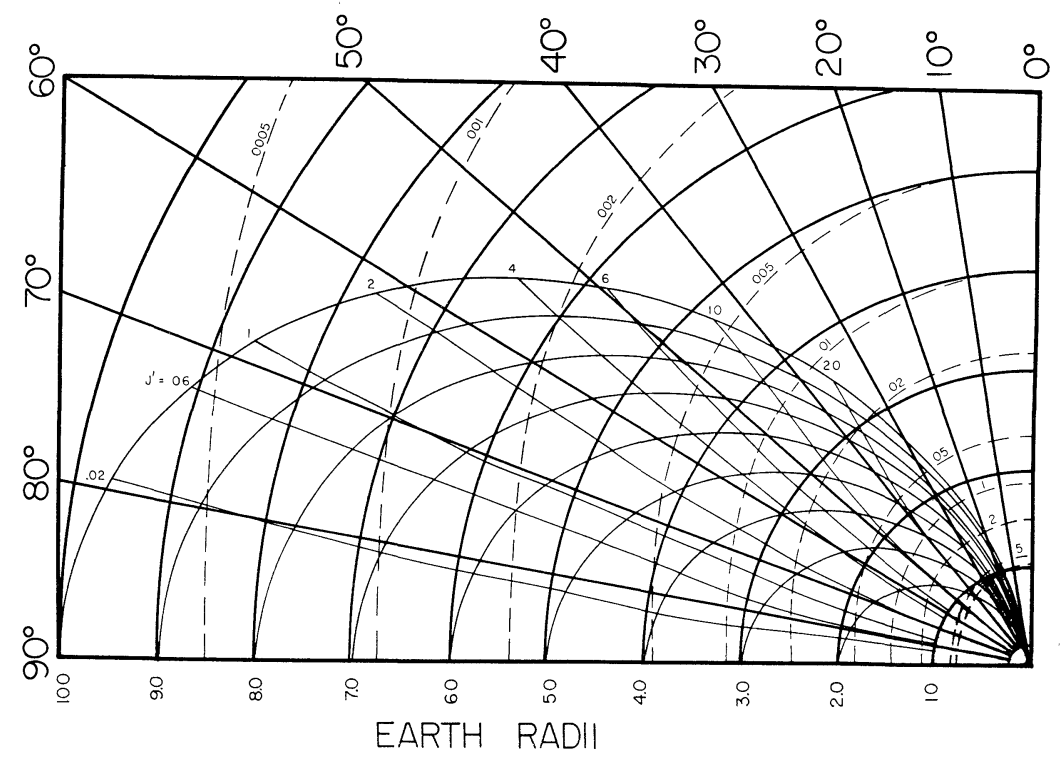

Fig. 1 Dipole field of the earth, showing lines of force, contours of constant field strength, and contours of constant $J^{\prime}$. Units of $J^{\prime}$ are (earth radii) $\times$ (gauss $)^{1 / 2}$. As a particle gains or loses kinetic energy its mirror point moves inward or outward along a contour (i.e., surface) of constant $J^{\prime}$ and the kinetic energy varies directly as the mirror field strength, $B_{m}$.

related to its radial distance, $r$, from the dipole and to its initial kinetic energy and radial position :

$$
W=W_{0}\left(B_{m} / B_{m_{0}}\right)=W_{0}\left(r_{0} / r\right)^{3}=W_{0}\left(L_{0} / L\right)^{3}
$$

Here the subscript, $o$, indicates initial values, and the radial position, $r$, has been replaced by $L$, the equatorial radius (in earth radii) of the line of force on which the particle is located. In a conservative electric field derived from a pontential, $V$, the total energy of a drifting particle, $K=W+e V$ ( $e$ represents the particle's charge) is constant. If the potential, $V=V$ $(L, \phi)$, is known as a function of $L$ and $\phi$ ( $\phi=$ the longitude measured from a suitable reference line such as the earth-sun line), the particle's location, $L=L(\phi)$, is simply obtained by rewriting (4) as :

$$
\frac{L}{L^{0}}=\left[1+\frac{e\left(V_{0}-V\right)}{W_{0}}\right]^{-1 / 3}
$$

When the particle does not mirror initially in the equatorial plane of the dipole, the relation $W / W_{0}=B_{m} / B_{m_{0}}$, expressing conservation of the magnetic moment still applies but it no longer provides the obvious simple relation (.ie., $\left.W \propto(1 / L)^{3}\right)$ detween $W$ and $L$ that was found in equation (4). One can, of course, refer to Figure 1 to determine how $B_{m}$ (and thus, $W$ ) vary with $L$ as a particle moves along a constant $J^{\prime}$ surface. However, we shall see that this is not necessary because a simple empirical relationship between $B_{m}$ and $L$ still applies for particles mirroring outside the equatorial plane.

To show what relationship does hold between $W\left(\right.$ or $\left.B_{m}\right)$ and $L$ with conservation of $M$ 
and $J^{\prime}$ in a dipole field, there is plotted, in Figure 2 the ratio $\left(B_{m}(L) / B_{m}(10)\right)^{1 / 3}$ versus the ratio $(10 / L)$ using corresponding values of $B_{m}(L)$ and $L$ picked along curves of constant $J^{\prime}$ in Figure 1. The individual points are not shown, but they were found to lie quite precisely along the straight lines shown in the log-log plot. The line for $J^{\prime}=0$ has, of course, unit slope in this representation. The slopes of the other lines are less than one and decrease with increasing $J^{\prime}$, showing that $B_{m}$ forparticles mirroring at large angles above and below the equator varies less strongly with $L$ than it does for particles mirroring near the equator. The slope, $S$, of each line Figure 2 is :

$$
S=\log \left(B_{m}(L) / B_{m}(10)\right)^{1 / 3} / \log (10 / L)
$$

and it is easily shown from this that, in general,

$$
B_{m a} / B_{m b}=\left(L_{b} / L_{a}\right)^{3 S}
$$

where $a$ and $b$ refer to two different lines of force identified by their equatorial radii $L_{a}$ and $L_{b}$. The parameter, $S$, is a function only of $J^{\prime}$, and, thus, is a constant, as is $J^{\prime}$, determined by the initial conditions of the particles' motion.

Figure 3 shows values of the exponent, $\gamma \equiv 3 S$ plotted against $J^{\prime}$. It is noted that $\gamma$ approaches the value 3 for very small $J^{\prime}$ and appears to approach the value 2 for very large $J^{\prime}$. Indeed, $\gamma$ should approach 2 for very large $J^{\prime}$ because, in that limit, when $B_{m} \gg B_{e q}$, the in-

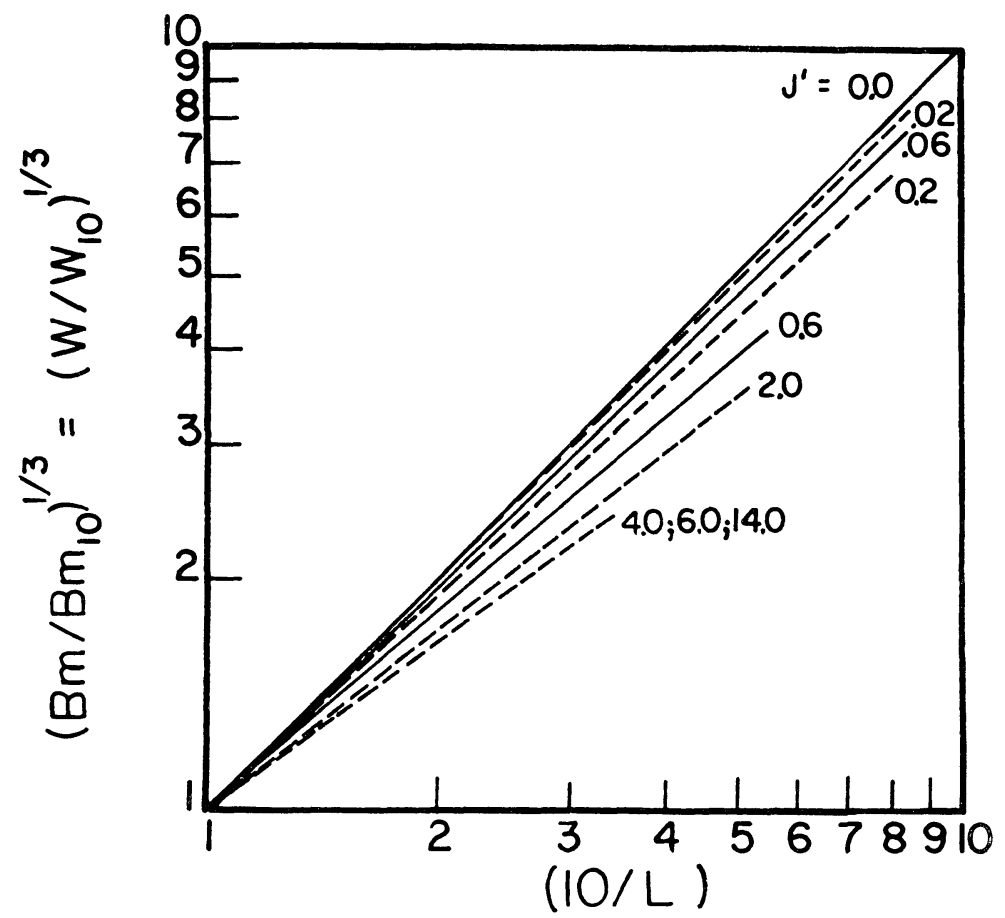

Fig. 2 The ratio, $\left(B_{m}(L) / B_{m}(10)\right)^{1 / 3}$ plotted versus $(10 / L)$ for various values of $J^{\prime} . B_{m}(10)$ is the mirror field strength of a particle, having a given $J^{\prime}$, on the $L=10$ line. $B_{m}(L)$ is the mirror field strength of a particle having the same $J^{\prime}$ but on another line $L$. The units of $I^{\prime}$ are (earth-radii) $\times\left(\right.$ gauss $\left.^{1 / 2}\right)$. 


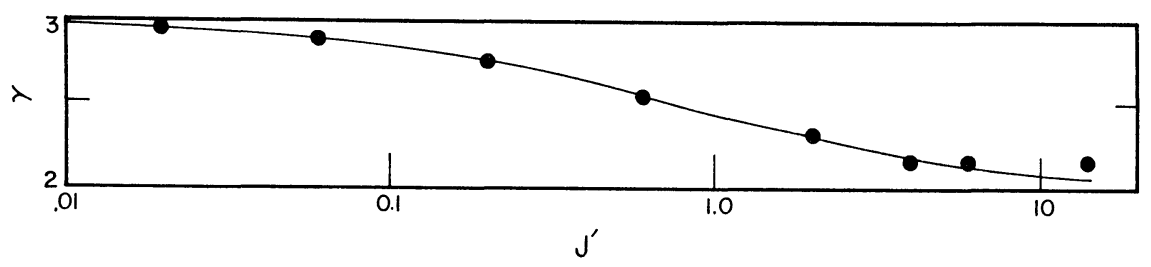

Fig. 3 The exponent, $r \equiv 3 S$ plotted against $J^{\prime} . \quad \gamma$ has value 3 for $J^{\prime}=0$ (i.e., particles mirroring in the equatorial plane) and approaches 2 for large $J^{\prime}$ (i.e., particles mirroring at high latitudes).

tegral $\oint\left(1-B / B_{m}\right)^{1 / 2}$ ds becomes proportional to $L$ and then, to keep $J^{\prime}$ constant with varying $L, B_{m}$ must become proportional to $L^{-2}$.* The curve of Figure 3 , together with knowledge of the initial mirror point (and, thus, the value, $J^{\prime}$ ) for a particle, permits one to use equation (5) in the following form to trace a particle's path:

$$
\frac{L}{L_{0}}=\left[1+\frac{e\left(V_{0}-V\right)}{W_{0}}\right]^{-1 / r}
$$

It is clear from equation (8) that particles, initially in a single tube of force, will generally follow different paths, the path of each particle depending upon its initial kinetic energy and mirror point in the tube of force.

In summary, then, equation (8) enables one to determine the path of a drifting particle (that is, $L$ versus $\phi$ ) in a dipole magnetic field (in which the lines of force are equipotentials) when the charge of the particle, its initial kinetic energy, and initial mirror point are known and when the distribution of electric potential among the field lines is stated. When the drift path is thus established, $B_{m}$ (and thus the kinetic energy) at each point is known, since $B_{m} / B_{m_{0}}=\left(L_{0} / L\right)^{\gamma}$, and the mirror altitude is determined from the mirror field strength $B_{m}$.

\section{Illustration of Use of Foregoing Results}

Maeda [1964] presents the world-wide distribution of the electrostatic field in the $E$-region (as deduced from geomagnetic data) in potential form as follows :

$$
V(a, \theta, \phi)=a \sum_{n} \sum_{m}\left(A_{n}^{m} \cos m \phi+B{ }_{n}^{m} \sin m \phi\right) P_{n}^{m}(\cos \theta)
$$

where $\theta=$ colatitude

$\phi=$ longitude (or local time in angular measure, reckoned from midnight)

$a=$ radius of ionospheric currentt sheet $\left(6.48 \times 10^{8} \mathrm{~cm}\right)$

$\stackrel{m}{P}(\cos \theta)=$ Schmidt's Functions [see Chapman and Bartels, 1962].

Maeda lists the values of the harmonic coefficients $A$ and $B$ for quiet days. We have used his values in equation (9) to compute $V(a, \theta, \phi)$ for every five degrees of longitude, $\phi$, and for values of $\theta$ corresponding to $L$-values from $1 R_{e}$ to $10 R_{e}$ in $0.2 R_{e}$ increments. ( $L$ is related to $\theta$ by $\sin ^{2} \theta=1 / L$.) This potential distribution, projected along lines of force into the equa-

\footnotetext{
* I wish to thank Mr. Harold E. Taylor for pointing out the logic of the lower limiting value, 2, of the exponent, $r$. Axford and Hines [1961] reached an analogous conclusion by considering the process of energization of low-energy particles to be one of adiabatic compression of tubes of force.
} 
torial plane of the dipole field, is sketched in Figure 4. One sees a relative high potential near midnight and a low potential near noon. Since the $E$-region rotates with the earth (at least at low latitudes) one must add the co-rotational electric field given by the potenital:

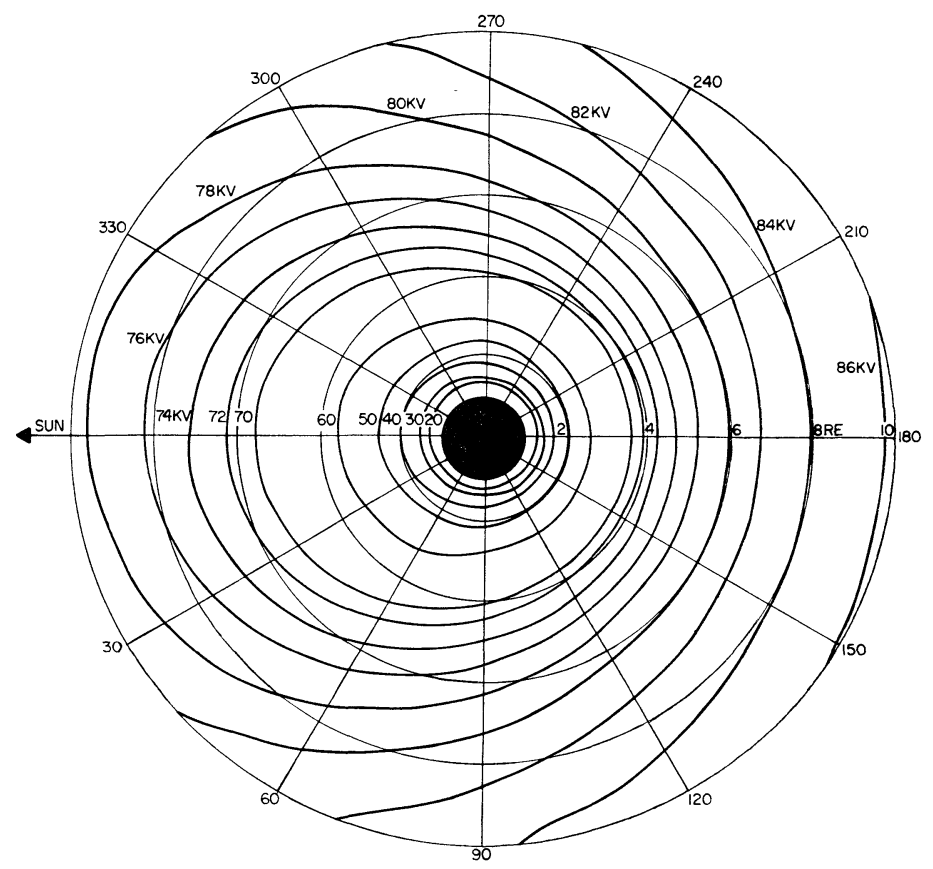

Fig. 4 Potential distribution in equatorial plane of dipole field as calculated from data of Maeda [1964].

$$
V=\frac{M_{E} \omega_{E}}{10^{8} a}\left(1-\frac{1}{L}\right)(\text { volts })
$$

where $M_{E}=$ magnetic moment of earth $\left(8.1 \times 10^{25}\right.$ gauss-cm $\left.{ }^{3}\right)$

$\omega_{E}=$ angular velocity of earth

$a=$ radius of ionospheric current sheet.

This becomes

$$
V=92\left(1-\frac{1}{L}\right) \text { kilovolts }
$$

The sum of the two potentials, projected into the equatorial plane of the dipole field, is sketched in Figure 5.

To illustrate the use of the invariants of particle motion in calculating particle drift paths, we have used equation (8), together with the computed values of potential depicted in Figure 5 to calculate the path of a $1 \mathrm{keV}$ electron initially mirroring on the $L=3$ line at midnight and an altitude of $160 \mathrm{~km}(B \sim 0.5$ gauss.) For this initial mirror point, our calculations (from which the $J^{\prime}$ curves in Figure 1 were sketched) show that $J^{\prime}=4.0$. Therefore, by Figure 3 , $\gamma \sim 2.2$. Figure 5 shows that the potential of the initial line is $\sim 65 \mathrm{kV}$. (The precise value taken from the tables of computed values is 65.288 kilovolts.) The determination of the path 


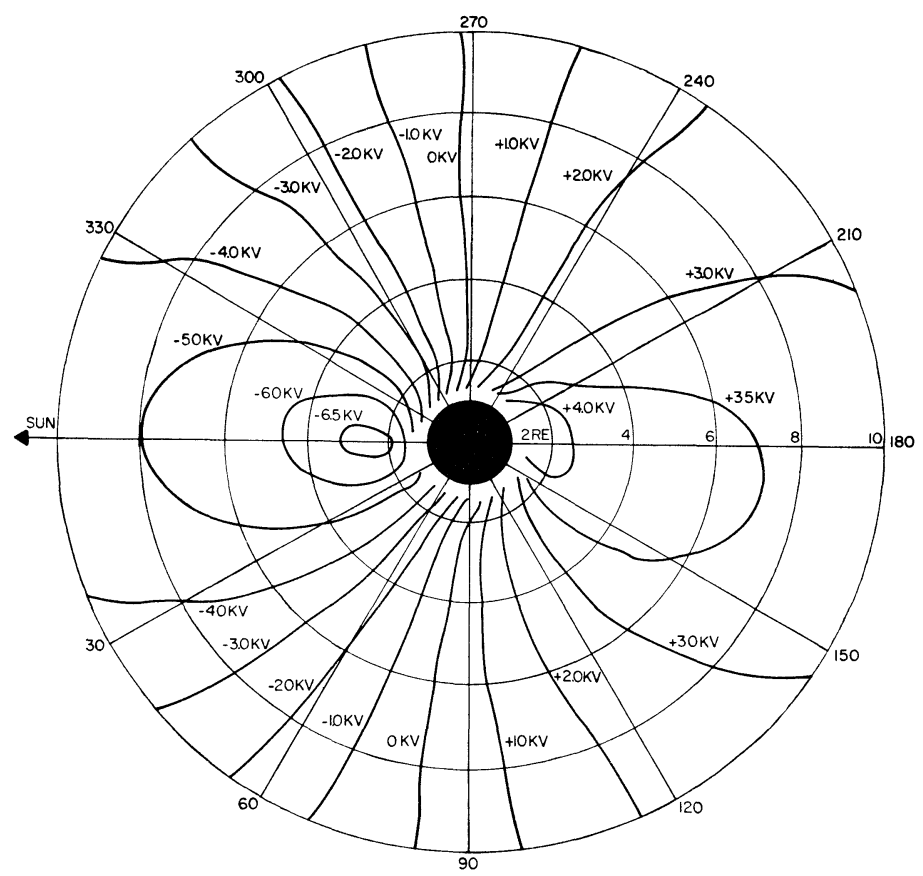

Fig. 5 Potential distribution in equatorial plane of dipole field. The co-rotation potential is included.

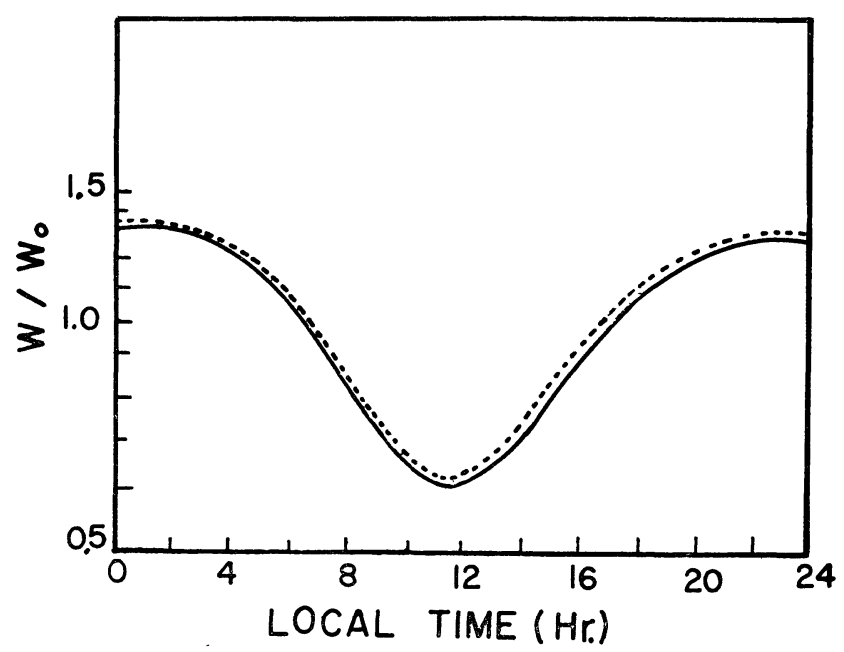

Fig. 6 Variation of kinetic energy of an electron with local time. Solid curve represents electron initially mirroring with 1 $\mathrm{keV}$ kinetic energy at $160 \mathrm{~km}$ altitude on the $L=3$ line at midnight; calculation done by methods described in this paper. Dashed curve is taken from Figure 4 of Maeda [1964] and applies to a $1 \mathrm{keV}$ electron on $L=3.09$ line.

consists? simply in choosing values of $L$ (i.e., $L=2.8,2.6$, etc.) from those for which $V$ was computed, taking the ratio of each to $L_{0}$ (which was 3.0) and solving for the corresponding values of $V$. Then, since $V$ is known, for each $L$, as a function of $\phi$, one finds the longitude 
$(\phi)$ at which the particle crosses each $L$-shell.

These particular starting conditions were chosen in order to provide a comparison of our results with those of Maeda who presented (his Figure 4) the kinetic energy variation of a $1 \mathrm{keV}$ electron with very similar initial conditions (initially mirroring at $120 \mathrm{~km}$ altitude on the $L=3.09$ line at miduight). Maeda's method of calculation involved a numerical integration of the work done by the electric field depicted in Figure 4 upon the drifting electron, presumably as the rotating magnetosphere carried it through this potential pattern.

Figure 6 shows the kinetic energy versus local time as calculated by Maeda (dashed curve) and by us (solid curve). The two curves are quite similar. This is not surprising, as they simply represent the results of doing the same calculation two different ways; this particular calculation has been presented simply to illustrate the method described in the foregoing sections.

\section{Conclusions}

Use can be made of the fact of conservation of the first and second adiabatic invariants in determining paths of particles, not only in a pure magnetic field, but also in combined magnetic and electric fields. This becomes particularly attractive when the magnetic lines of force are equipotentials of the electric field, a situation which probably prevails widely in the magnetosphere. If the magnetic field is that of a dipole the tracing of paths becomes very simple because the mirror field strength, $B_{m}$, and thus the kinetic energy, $W$, depends explicitly upon $L$. The nature of this dependence is a function only of the initial mirror position of the particle and we have found that, to very good approximation,

$$
\frac{W_{a}}{W_{b}}=\frac{B_{\mathrm{ma}}}{B_{\mathrm{mb}}}=\left(\frac{L_{b}}{L_{a}}\right)^{r}
$$

where $\gamma$, determined empirically, ranges from 3.0 for particles moving only in the equatorial plane (i.e., $J^{\prime}=0$ ) to $\sim 2$ for particles mirroring at high latitudes (i.e., large $J^{\prime}$ ).

\section{References}

Axford W. I., and C.O. Hines, A unifying theory of high latitude geophysical phenomena and geomagnetic storms, Can. J. Phys., 39, 1433, 1961.

Chapman, S., and J. Bartels, Geomagnetism, Vol. II, p. 611, Oxford University Press, 1962.

Maeda, H., Electric fields in the magnetosphere associated with daily geomagnetic variations and their effect on trapped particles, J. Atm. and Terr. Phys., 26, 1133, 1964.

McIlwain, C.E., Coordinates for mapping the distribution of magnetically trapped particles, J. Geophys. Res., 66, 3681, 1961.

Northrop, T.G., and E. Teller, Stability of the adiabatic motion of charged particles in the earth's field, Phys. Rev., 117, 215-225, 1960. 